\title{
Ueber die Darstellung der Curven durch Krümmung und Torsion.
}

(Von Herrn R. Hoppe.)

Sind die Krümmung und Torsion einer Curve als Functionen des Bogens gegeben, so ist ihre Gestalt unabhängig von ihrer Lage vollständig bestimmt. Um daraus ihre Gleichungen in rechtwinkligen Coordinaten zu finden, hat man sechs Integrationen zu vollziehen, entsprechend den sechs Constanten, welche ihre Lage im Raume bestimmen. Dies ist in zwei Fällen leicht: 1) wenn die Torsion $=0$ ist; 2) wenn sie ein constantes Verhältniss zur Krümmung hat. Ausserdem giebt es noch manche sehr einfache Relationen zwischen beiden Variabeln, welche die Integration zulassen. Obgleich die allgemeine Lösung der Aufgabe nicht wohl möglich ist, so scheinen mir doch einige Reductionen, deren sie fähig ist, an sich von Interesse zu sein, namentlich insofern daraus ersichtlich wird, wovon die Integrabilität im speciellen Falle abhängt. Ich erlaube mir, von den Benennungen Gebrauch zu machen, welche ich in meinem letzten Aufsatze (Band 58, p. 374) erklärt habe.

I.

Bezeichnen $\tau$ und $\vartheta$ den Krümmungs - und Torsionswinkel einer Curve (d. i. die Integrale der Contingenzwinkel der Tangenten und Osculationsebenen); $u, v, w$ die cos. der Winkel, welche die Tangente, Pollinie und Hauptnormale mit irgend einer Axe bilden; so ist wegen der senkrechten Lage dieser Geraden:

$$
\text { (1.) } u^{2}+v^{2}+w^{2}=1
$$

und gemäss bekannten Formeln:

$$
\partial u=w \partial \tau ; \quad \partial v=-w \partial \vartheta ; \quad \partial w=v \partial \vartheta-u \partial \tau,
$$

woraus hervorgeht:

$$
\boldsymbol{v}=\frac{\partial \boldsymbol{\tau}}{\partial \boldsymbol{\vartheta}}\left(u+\frac{\partial^{2} u}{\partial \boldsymbol{\tau}^{2}}\right) ; \quad \boldsymbol{u}=\frac{\partial \vartheta}{\partial \boldsymbol{\tau}}\left(\boldsymbol{v}+\frac{\partial^{2} v}{\partial \vartheta^{2}}\right)
$$

Jenachdem man nun $v$ oder $u$ nebst $v$ aus Gleichung (1.) eliminirt, erhält man

$$
\text { (4.) } \quad\left(\frac{\partial \vartheta}{\partial \tau}\right)^{2}\left(v+\frac{\partial^{2} v}{\partial \vartheta^{2}}\right)^{2}+v^{2}+\left(\frac{\partial v}{\partial \vartheta}\right)^{2}=1 \text {. }
$$

Hiernach steht jede Curve im Allgemeinen mit einer zweiten in der gegenseitigen Beziehung, dass der Krümmungswinkel der einen der Torsionswinkel der anderen, und die Tangente der einen parallel der Pollinie der anderen 
ist. Die Integration einer der vorstehenden Gleichungen erledigt somit die zwei Fälle gegebener Functionen

$$
\varphi(\tau, \vartheta)=0, \quad \varphi(\vartheta, \tau)=0
$$

zu gleicher Zeit. Das Folgende mag sich deshalb auf Betrachtung der Gleichung (3.) beschränken. Demgemäss soll $\tau$ als unabhängige Variable betrachtet werden, und die Accente Differentialquotienten nach derselben bezeichnen.

Nach einmaliger Differentiation und Ausscheidung des Factors $u+u^{\prime \prime}$ giebt' Gleichung (3.):

$$
\text { (5.) } \quad \boldsymbol{u}^{\prime \prime \prime}+\left(\boldsymbol{1}+\boldsymbol{\vartheta}^{\prime 2}\right) \boldsymbol{u}^{\prime}-\frac{\boldsymbol{\vartheta}^{\prime \prime}}{\boldsymbol{\vartheta}^{\prime \prime}}\left(\boldsymbol{u}+\boldsymbol{u}^{\prime \prime}\right)=0 \text {. }
$$

Das vollständige Integral dieser linearen Gleichung hat die Form:

$$
u=a u_{1}+b u_{2}+c u_{3},
$$

doch sind die Constanten $a, b, c$ einer Relation unterworfen, die sich durch Einführung in Gleichung (3.) ergiebt. Wählt man dam drei Systeme der $a, b, c$ so, dass die Quadratsumme der drei entsprechenden Werthe von $u=1$ wird, so stellen diese die cos. der Richtungswinkel der Tangente gegen drei rechtwinklige Axen dar, und geben nach Multiplication mit dem Differential des in $\tau$ ausgedrückten Bogens und folgender Integration die Coordinaten als Functionen von $\tau$.

Da hiernach die Lösung der Aufgabe vollendet ist, ehe der Bogen in Rechnung kommt, so erhält man durch Integration der Gleichung (3.) für jede specielle Relation zwischen $\tau$ und $\vartheta$ eine Classe von Curven, deren gemeinsames Merkmal eben jene Relation ist, während die Funclion, die den Bogen beliebig in $\tau$ ausdrückt, die Verschiedenheit ihrer Gestalt bedingt. Die Abweichung kann hier allein in der Ausdehnung der einzelnen Bogenelemente stattinden, so dass sich jede Klasse dem Auge leicht als zusammengehörig darstellt.

\section{II.}

Zur weiteren Transformation der Differentialgleichung selze man:

$$
u=q \int \frac{\partial \tau}{q^{2}} \sqrt{q^{2}+q^{\prime 2}}
$$

woraus durch Auflösung nach $q$ hervorgeht:

$$
q=\sqrt{1-u^{i}} e^{i \int \frac{\partial_{c}}{1-u^{2}} \sqrt{1-u^{2}-u^{2}}} .
$$

Dann geht Gleichung (3.) nach Ausscheidung des Faclors

über in

$$
\left(\frac{q^{\prime}}{q \sqrt{q^{2}+q^{\prime 2}}}+\int \frac{\partial \tau}{q^{2}} \sqrt{q^{2}+q^{\prime 2}}\right)^{2}
$$


184 Hoppe, über die Darstellung der Curven durch Krïmmung und Torsion.

$$
\text { (6.) }\left(\frac{q+q^{\prime \prime}}{\vartheta^{\prime}}\right)^{2}+q^{2}+q^{\prime 2}=0 \text {. }
$$

Dieselbe Gleichung würde man auch als besonderes Integral der Gleichung (5.) erhalten, wenn man die Integrationsconstante, welche gemäss Gleichung (3.) den Werth 1 haben muss, $=0$, und den entsprechenden Werth von $u=q$ setzte. Die vorstehende Herleitung zeigt, in welcher Beziehung die wirklichen Wurzeln der Gleichung (3.) zu diesen unbrauchbaren der Gleichung (5.) stehen.

Ferner sei

woraus umgekehrt

$$
q=r e^{-\int \frac{r d r}{4 r^{\prime}}}
$$

$$
r=\sqrt{q} e^{-\frac{1}{2} \int \frac{\partial q}{q} \sqrt{q^{2}+q^{\prime 2}}} .
$$

Dann geht Gleichung (6.) nach Ausscheidung des Factors

$$
q^{2}\left(1+\frac{r^{2}}{4 r^{\prime 2}}\right)^{2}
$$

über in

$$
\frac{1}{\vartheta^{\prime 2}}\left(\frac{r^{\prime \prime}}{r}+\frac{1}{4}\right)^{2}+\frac{r^{\prime 2}}{r^{2}}=0
$$

woraus

(7.) $r^{\prime \prime} \pm i \vartheta^{\prime} r^{\prime}+\frac{1}{4} r=0$.

Die möglichen Transformationen dieser Gleichung sind bekannt. Die einfachste Form erhält sie durch die Substilution:

nämlich die folgende:

$$
r=\frac{p}{\sigma}, \quad \tau=2 \int \frac{\partial \sigma}{\sigma^{2}} e^{ \pm i \vartheta}
$$

$$
\frac{\partial^{2} p}{\partial \sigma^{2}}+\frac{p}{\sigma^{4}} e^{ \pm 2 i \vartheta}=0
$$

III.

Ist eine Speciallösung der Gleichung (3.) bekannt, so ist die vollständige Lösung der Aufgabe durch ihre Zurückführung auf eine lineare Gleichung zweiter Ordnung verbürgt. Doch kann man auf weit kürzerem Wege dazu gelangen, als durch Vermittlung der Gleichung (7.). Sind nämlich

$$
u=\cos \varphi \cos \psi, \quad v=\sin \varphi \cos \psi, \quad w=\sin \psi
$$

die cos. der Richtungswinkel der Tangente; ist ferner $w$ als Function von $\tau$ bekannt, und man setzt

$$
\psi^{\prime}=\sin \omega \text {, }
$$

so sind auch $\psi$ und $\omega$ bekannt. Für die cos. der Richtungswinkel der Hauptnormale findet man durch eine Differentiation folgende Werthe: 
Hoppe, über die Darstellung der Curven durch Krümmung und Torsion. 185

$$
\begin{aligned}
& u^{\prime}=-\varphi^{\prime} \sin \varphi \cos \psi-\cos \varphi \sin \psi \sin \omega, \\
& \boldsymbol{v}^{\prime}=\varphi^{\prime} \cos \varphi \cos \psi-\sin \varphi \sin \psi \sin \omega, \\
& \boldsymbol{w}^{\prime}=\cos \psi \sin \omega
\end{aligned}
$$

deren Quadratsumme ist:

$$
1=\varphi^{\prime 2} \cos ^{2} \psi+\sin ^{2} \omega .
$$

Durch den hieraus gewonnenen Werth

sind $u, v$ und demzufolge

$$
\varphi=\int \frac{\cos \omega}{\cos \psi} \partial \tau
$$

$$
x=\int u \partial s, \quad y=\int v \dot{\delta} s, \quad z=\int w \partial s
$$

als Functionen von $\tau$ bekannt. Durch eine Orthogonalsubstitution lassen sich die fehlenden Integrationsconstanten, deren zwei im vollständigen Werthe von $\psi$, und eine in $\varphi$ enthalten sein würden, leicht herstellen.

Die mittelst Gleichung (7.) sich ergebenden Ausdrücke von $u, v$ sind weit complicirter als die soeben abgeleiteten; die Identität beider möchte überdiess wohl auf anderem Wege nicht in der Kürze nachzuweisen sein.

IV.

Ich füge noch die Lösungen für vier der einfachsten Fälle in ihrer einfachsten Specialform bei. Durch $l, m, n$ seien die cos. der Richtungswinkel der Pollinie bezeichnet.

1) Ist $\vartheta^{\prime}=0$, so ist wie bekannt

$$
\begin{array}{lll}
u=\cos \tau, & v=\sin \tau, & w=0, \\
u^{\prime}=-\sin \tau, & v^{\prime}=\cos \tau, & w^{\prime}=0, \\
l=0, & m=0, & n=1 .
\end{array}
$$

2) Ist $\vartheta^{\prime}=\operatorname{tg} \lambda$ constant, so geht Gleichung (5.) über in

$$
u^{\prime \prime \prime} \cos ^{2} \lambda+u^{\prime}=0 \text {. }
$$

Bestimmt man den constanten Factor des Integrals gemäss Gleichung (3.), so ergiebt sich:

$$
\begin{array}{lll}
u=\cos \lambda \cos \frac{\tau}{\cos \lambda}, & v=\cos \lambda \sin \frac{\tau}{\cos \lambda}, & w=\sin \lambda, \\
u^{\prime}=-\sin \frac{\tau}{\cos \lambda}, & v^{\prime}=\cos \frac{\tau}{\cos \lambda,}, & w^{\prime}=0, \\
l=-\sin \lambda \cos \frac{\tau}{\cos \lambda}, & m=-\sin \lambda \sin \frac{\tau}{\cos \lambda}, & n=\cos \lambda .
\end{array}
$$

3) Setzt man $w=\frac{\tau}{\alpha}$, so ergiebt sich die Relation:

$$
x^{2}+\vartheta^{2}=\alpha^{2}-1
$$

welche erfüllt wird durch die Werthe: 
186 Hoppe, wber die Darstellung der Curven durck Krümmong und Torsion.

$$
\tau=\sqrt{\alpha^{2}-1} \sin \lambda ; \quad \vartheta=-\sqrt{\alpha^{2}-1} \cos \lambda,
$$

und man findet nach der im vorigen Abschnitte beschriebenen Methode leicht folgende Ausdrücke:

$$
\begin{aligned}
& u=\frac{1}{\alpha} \sin \alpha \lambda \sin \lambda+\cos \alpha \lambda \cos \lambda, \\
& v=-\frac{1}{\alpha} \cos \alpha \lambda \sin \lambda+\sin \alpha \lambda \cos \lambda, \\
& w=\frac{\sqrt{\alpha^{2}-1}}{\alpha} \sin \lambda, \\
& u^{\prime}=-\frac{\sqrt{\alpha^{2}-1}}{\alpha} \sin \alpha \lambda, \quad v^{\prime}=\frac{\sqrt{\alpha^{2}-1}}{\alpha} \cos \alpha \lambda, \quad w^{\prime}=\frac{1}{\alpha} \\
& l=\frac{1}{\alpha} \sin \alpha \lambda \cos \lambda-\cos \alpha \lambda \sin \lambda, \\
& m=-\frac{1}{\alpha} \cos \alpha \lambda \cos \lambda-\sin \alpha \lambda \sin \lambda, \\
& n=\frac{\sqrt{\alpha^{2}-1}}{\alpha} \cos \lambda .
\end{aligned}
$$

4) Setat man $\omega=\psi$, so wird

$$
\varphi=\tau, \quad \vartheta=2 \psi, \quad \operatorname{tg} \frac{\vartheta}{4}=e^{\tau},
$$

daher

$$
\begin{aligned}
& u=\cos \tau \cos \frac{\vartheta}{2}, \quad v=\sin \tau \cos \frac{\vartheta}{2}, \quad w=\sin \frac{\vartheta}{2}, \\
& u^{\prime}=-\sin \tau \cos \frac{\vartheta}{2}-\cos \tau \sin ^{2} \frac{\vartheta}{2}, \\
& v^{\prime}=\cos \tau \cos \frac{\vartheta}{2}-\sin \tau \sin ^{2} \frac{\vartheta}{2}, \\
& w^{\prime}=\sin \frac{\vartheta}{2} \cos \frac{\vartheta}{2}, \\
& l=\left(\sin \tau-\cos \tau \cos \frac{\vartheta}{2}\right) \sin \frac{\vartheta}{2}, \\
& m=-\left(\cos \tau+\sin \tau \cos \frac{\vartheta}{2}\right) \sin \frac{\vartheta}{2}, \\
& n=\cos ^{2} \frac{\vartheta}{2} . \quad V .
\end{aligned}
$$

Geometrisch lässt sich das Problem auf folgende Weise reduciren. Beschreibt man mit der Linieneinheit als Radius eine Kugel um den Anfangspunkt der Coordinaten, und zieht mit der Tangente der Curve einerseits mit ihrer Pollinie andererseits zwei parallele Radien, so beschreiben diese auf der Kugelfläche zwei Curven, die einander parallel sind, weil ter laufende Punkt 
einer jeden auf der entsprechenden Normalebene der anderen liegt. Durch Vertauschung von Krümmung und Torsion der Urcurve geht die eine jener sphärischen Curven in die andere über. Es wird hinreichen, die Beziehung der ersten zur Urcurve zu untersuchen.

Unterscheidet man die Elemente dieser sphärischen Curve durch den Index 1, so sind ihre Gleichungen:

dies differentiirt giebt:

$$
x_{1}=u, \quad y_{1}=v, \quad z_{1}=v,
$$

$$
u_{1} \partial s_{1}=u^{\prime} \partial \tau, \quad v_{1} \partial s_{1}=v^{\prime} \partial \tau, \quad w_{1} \partial s_{1}=w^{\prime} \partial \tau .
$$

Durch Bildung der Quadratsumme zeigt sich, dass

$$
\text { (8.) } \quad \begin{cases}s_{1}=\tau, \\ u_{1}=u^{\prime}, & v_{1}=v^{\prime}, \quad w_{1}=w^{\prime}\end{cases}
$$

ist. Eine zweite Differentiation ergiebt:

$$
u_{1}^{\prime} \partial \tau_{1}=(l \operatorname{tg} \lambda-u) \partial \tau
$$

nebst zwei analogen Gleichungen, aus deren Quadratsumme erhellt, dass

$$
\text { (9.) }\left\{\begin{array}{l}
\cos \lambda \partial \tau_{1}=\partial \tau, \\
u_{1}^{\prime}=l \sin \lambda-u \cos \lambda, \quad \text { etc. }
\end{array}\right.
$$

und eine dritte Differentiation mit Zuziehung der Gleichungen (9.) giebt

$$
l_{1} \vartheta_{1}^{\prime}-u_{1}=\lambda^{\prime}(l \cos \lambda+u \sin \lambda) \cos \lambda-u^{\prime},
$$

oder nach den Gleichungen (8.)

$$
l_{1} \vartheta_{1}^{\prime}=\lambda^{\prime}(l \cos \lambda+u \sin \lambda) \cos \lambda
$$

nebst zwei analogen Gleichungen, deren Quadratsumme ergiebt

oder nach Gleichung (9.)

$$
\vartheta_{1}^{\prime}=\lambda^{\prime} \cos \lambda
$$

$$
\partial \vartheta_{1}=\partial \lambda \text {. }
$$

Ferner lässt sich Gleichung (9.) auch schreiben:

$$
s_{1}^{\prime}=\cos \lambda \text {, }
$$

welche Grösse den Krümmungsradius der sphärischen Curve ausdrückt.

Es hat sich ergeben, dass der Bogen der sphärischen Curve den Krümmungswinkel, ihr Krümmungsradius den Cosinus der Krümmungsbreite, und ihr Torsionswinkel die Krümmungsbreite der Urcurve selbst darstellt. Ist nun 9 mithin auch $\lambda=\operatorname{arctg} \vartheta^{\prime}$ in $\tau$ gegeben, so ist auch der Krümmungsradius der sphärischen Curve als Function ihres Bogens bekannt. Die anfänglich gestellte Aufgabe ist demnach auf die zurückgeführt: eine sphärische Curve aus der Relation zwischen Krümmung und Bogen zu berechnen.

Berlin, den $5^{\text {ten }}$ Januar 1861. 